\title{
Complex and Dynamic Data Representation by Sonification
}

\author{
Maher CHEMSEDDINE ${ }^{1}$ and Monique NOIRHOMME-FRAITURE ${ }^{2}$ \\ ${ }^{1}$ FUNDP Institut d'Informatique, Belguim, maher.chemseddine@fundp.ac.be \\ ${ }^{2}$ FUNDP Institut d'Informatique, Belguim monique.noirhomme@fundp.ac.be
}

\begin{abstract}
So far, data representation has been based on visuals. The huge size of data verges on overuse of the visual capability. Thus, there is a need to reduce the almost exclusive use of visual techniques to represent data in order to increase our perception bandwidth. In this research, we aim to solve this problem by integrating the audio component. More precisely, we are interested in representing data using musical melodies. This paper presents a model for music elements based on human emotion, to express alert messages displayed by computer network monitoring.
\end{abstract}

Keywords: data representation, sonification, emotional music.

\section{Introduction:}

In dynamic and complex datasets, we have many parameters represented or modeled which make it difficult to distinguish those using only visual methods. The capability of looking at some dimensions while listening to others allows one to process more information at once and make better correlations. Also, auditory accompaniment clearly leads to an improved visual information reception.

Sonification (Herman, 2006) exploits our auditory channel perception. In contrast with the vocal interfaces, sonification uses no-speech audio to convey information. There are various methods of sonification, such as Auditory icons (Conversy, 2000), Earcons (André, 1993), Audification (Herman and al., 1999) and Parameters mapping (Conversy, 2000). However the domain of sonification is still seldom used in Human Computer Interaction/Interface.

In literature there have been many projects that have tried to match images and sound. Distinguish among these projects is VoiceInteractive (Peter, 1996), which allows transferring grayscale images to sound thanks to the Fourier transformations. However, the sound produced by this mapping consists of a wide range of non-significant frequency variations that have no meaning to human 
perception. In another project, Weather sonification (Flowers and al., 2001), the correlation is made between different types of data, such as air pressure and temperature, with musical instruments. Here, the pitch is proportional to the value of data. Using an orchestration of data, Charlie Cullen and Eugene Coyle (2004) have proposed to sonify a database of employers using this mapping between data and musical melodies. For example, they propose to match the attributes of musical instruments or rhythmic and pitches to a tuple (database record). In an application domain close to our topic, Maria Barra (2000) presented her thesis in a project named Personal WebMelody(Taria and al., 2001), which is a customizable system of sonification to monitor web servers that generates music through external sources (audio CD, MP3, and so on.). In this project the musical melodies are predefined in advance and are configurable by a webmaster.

Several studies have been conducted to try to find the best techniques to convey information. However, the problem of finding an appropriate correlation between data and sound is far from being solved. For some applications, the desire is to create realistic matches in the hope that they will be immediately "bindable", but also understandable.

The main purpose of this research is to design a sonification system that produces digital music from data in a meaningful and harmonic way. We intend to develop a prototype that will sonify networks traffic using music.

\section{Relation between music and emotion}

Music has an important role in attracting attention, transporting implicit and explicit messages, generating emotions and helping one retain information. These ties between music and emotion are part of the human psyche, primordial in nature. The psychologist Wilhelm Wundt (Population Research Laboratory, 2006) has described three dimensions of human emotion: valence, arousal, and potency. These dimensions are a subject of study in various fields of research in psychology, sociology and neurology.

The "Circumplex" by Russell (1989) (Schubert, 1999) is a classification of a human emotion within a two-dimensional model. It is limited to the two axes valence and arousal. The presentation of "Circumplex" suggests that the emotions happy and unhappy are opposed with respect to the axes of valence. The second dimension, arousal, measures the human activeness or passiveness in emotion.

In literature we see the work of Emery Schubert (1999), who summarized several scholarly studies in the field of musical emotion during the 19th and 20th centuries, proposing a model of the relationship between music elements and emotion. This two-dimensional emotional space based on the "circumplex" model and divided into 4 Quadrants, indicating a distribution into 8 clusters of human emotion (Figure 1)

Each cluster has its own musical element properties. In this work we limit ourselves to the following elements: articulation, vibrato, rhythm, meter, pitch, pitch range, mode, pitch combination, harmony, key and tempo. 


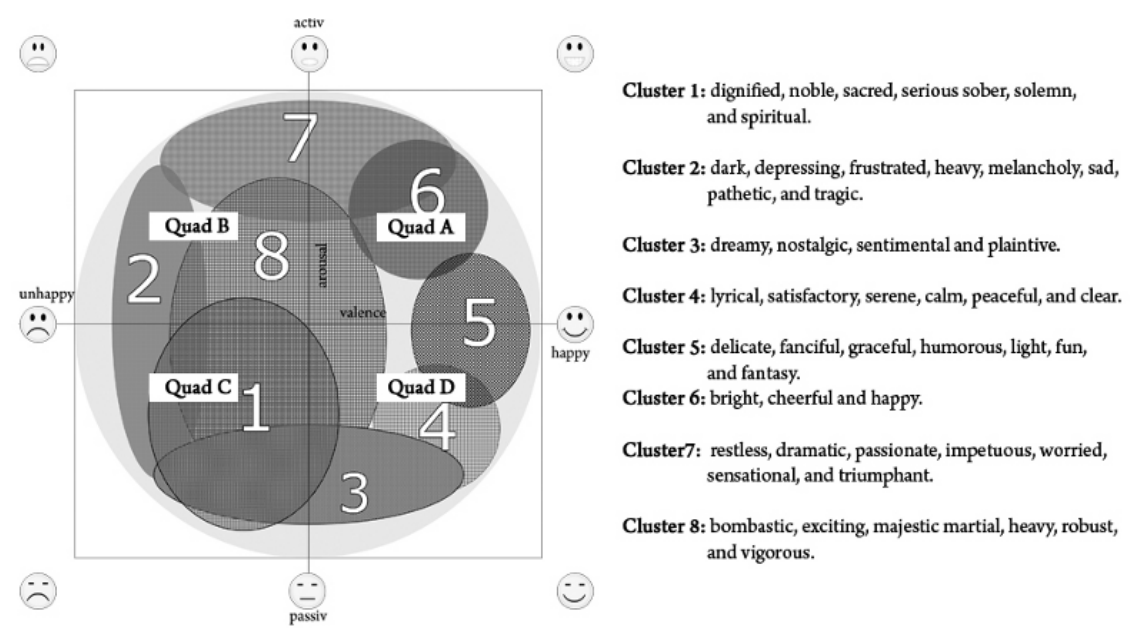

Figure 1 two-dimensional emotional space source: E. Shubert (1999).

\section{Data and emotion}

Our next step is to match data with emotion, and to do that we must first choose an application. We have decided to test our method with network traffic control and supervision. The main goal of this application is to implement a real time system of sonification, which gives the administrator information about network traffic through mobile phones, PDA and so on. The mapping of data created by the Algorithmic Composition technique (Alpern, 1995) transforms data into digital music, based on emotional music elements. We have started state of the art research within the fields of sonification and musical emotion, with the goal of creating an interlinking model.

\subsection{Data and emotion groups}

This study has been divided into two parts. The first part aims to find a relation between music elements (which are mainly melodic, harmonic and rhythmic) and information. We started by interviewing 6 network administrators, in order to classify different messages displayed by network monitoring tools. We distinguished two groups of messages, the first is called "Alert" and the second is called "Non-Alert". The "Alert" group is composed of Error, Warning, Critical, and Emergency messages. The "Non-Alert" is composed of Information, Notification and Debug. Administrators affirmed that their emotional state towards a problem in network depends primarily on three factors: 
- The impact of the problem on the user.

- The number of users infected by the problem.

- Their experience in dealing with such problem or similar ones.

The result of the interviews allowed us to have an idea about each message group's placement within the two-dimensional emotional space (Figure 2), finding that the representation of the group "Alert" occupies both quadrants B and C, and the group "Non-Alert" occupies quadrants A and D. According to that, clusters are distributed with respect to groups where:

- Group "Alert" occupies the clusters 1, 2, 3, 7, 8

- Group "Non-Alert" occupies the clusters 1, 3, 4, 5, 6, 7

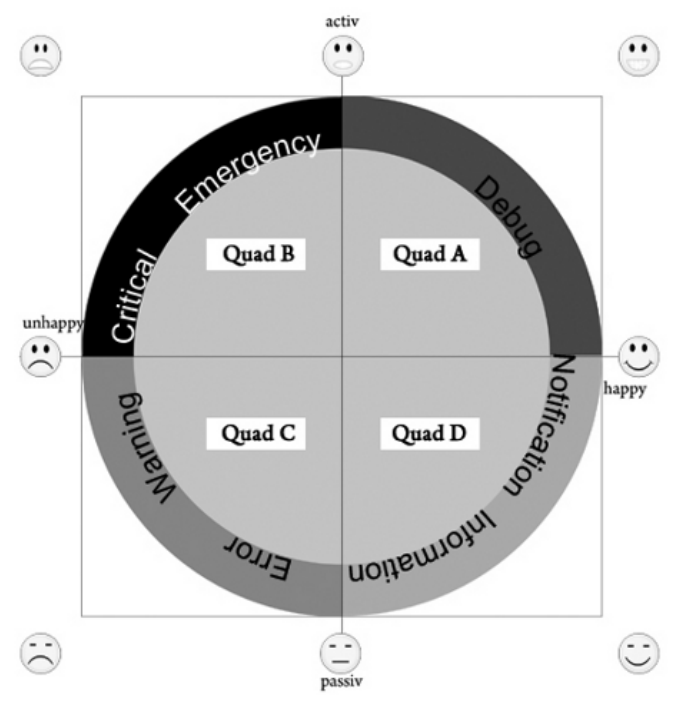

Figure 2 Classification of network message in two-dimensional emotional space.

A more precise placement will be concluded from a prepared survey planned to take place in the near future. Until then, with the help of this distribution along this two-dimensional emotional space and based on music elements of clusters (see section 2), we are still able to approach the different musical properties linked to each group.

Table 1. Example of music elements

\begin{tabular}{|c|c|c|c|c|c|}
\hline message & Articulation & meter & pitch & Mode & Combination \\
\hline Emergency & Legato & triple & $\begin{array}{l}\text { High } \\
\text { Low }\end{array}$ & minor & $\begin{array}{l}(1-2-3-4-5-6-5) /(5-6-5) /(5-1-2- \\
3) /(1-2-3-2-1) /(5-3-2-1) /(1-2- \\
3-4-5) /(1-2-3-2)\end{array}$ \\
\hline Warning & Legato & double & Low & minor & $\begin{array}{l}(5-4-3-2-1) /(8-7-6-5) / \\
(\text { descending chromatic) / (1-2-3- }\end{array}$ \\
\hline
\end{tabular}




\begin{tabular}{|l|l|l|l|l|l|}
\hline Error & & & & & $\begin{array}{l}2-1) /(5-3-2-1) /(1-2-3-4-5) /(1- \\
2-3-2)\end{array}$ \\
\hline Information & staccato & double & High & major & $\begin{array}{l}(5-1-2-3) /(1-2-3-4-5) /(5-6-5) / \\
(8-7-6-5) /(1-2-3-4-5-6-5) /(5-4- \\
\end{array}$ \\
& & & & \\
& & Low & & \\
\hline
\end{tabular}

\subsection{Online survey}

The second part consists of a multimodal (sound, graphic...) online survey via a website. Our goal here is to check our method, verify musical element in Table 2, and have a more precise classification of message subgroups. The human music composition used in the survey is based on music emotion element rules. Figure 3 represents a sample of musical partition of Error, Critical and Information messages (Chemseddine and Noirhomme, 2007). We have completed planning the experiment survey and selected 60 participants who are network administrators.

\section{Hypothesis of our study}

Our study is essentially based on "Western Music" and we have limited music elements in articulation; namely vibrato, rhythm, meter, pitch, pitch range, mode, pitch combination, key and tempo. Musicologists such as Byrd and Crawford (2001) (Kostek, 2005) argue that the most informative attributes in music are the melody and rhythm, allocating approximately 50\% for melody and $40 \%$ for rhythm. The remaining $10 \%$ consists of harmony, dynamics, and articulation. Also, we have chosen to arrange music with a piano timber.
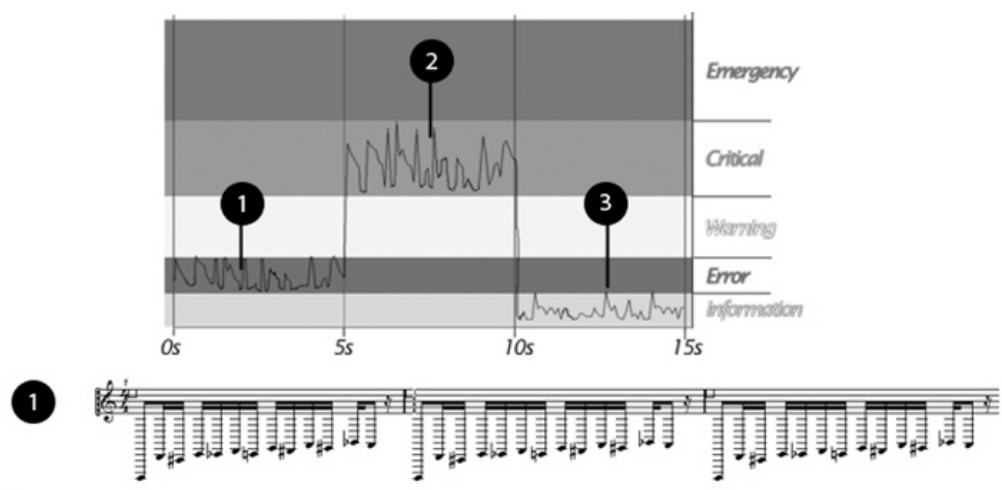

\section{(2)}
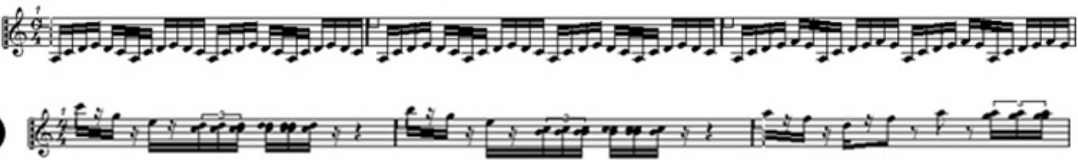

Figure 3 human music creations mapping graphic data 


\section{Conclusion and perspective}

The next part of our work aims to develop algorithms for music composition and to test our application with users. We believe that our system can be used in various fields such as in the industrial production in processes control, in the sonification of content, in video games, in monitoring different networks (roads, computer, telephony etc.) and in musicotherapy.

\section{References:}

Alpern, A..: Techniques for Algorithmic composition of Music, Hampshire College (1995).

André, S., Peter, C., and Edwards, D.N.: An Evaluation of Earcons for Use in Auditory HumanComputer Interfaces, University of York (1993).

Conversy, S. : Conception d'icônes auditives paramétrées pour les interfaces homme-machine, pp. 43-46 (2000).

Coyle, E., Cullen, C.: Orchestration within the Sonification of basic Data, Institute of Technology, Belfast (2004).

Chemseddine, M., Noirhomme, M.: Sound samples: http://netmelody.media19.be/samples.html (2007).

Flowers, J.H., Whitwer, L.E., Grafel, D.C. and Kotan, C.A.: Sonification of daily weather records: issues of Perception, attention and memory in design choices, University of Nebraska (2001).

Hermann, T., Ritter, H.: Listen to your Data: Model-Based Sonification for Data Analysis, University of Bielefeld (1999).

Hermann, T.: http://www.sonification.de/ (2006).

Kostek, B.: Perception-Based Data Processing in Acoustics, vol.3, p.30, Springer Heidelberg (2005).

Peter, B.L.: The Voice, http://www.seeingwithsound.com/javoice.htm (1996).

Population Research Laboratory: Valence and Arousal as Fundamental Dimensions of Emotional Experience (2006).

Schubert, E.: Measurement and time series analysis of emotion in music, Vol.1 (1999).

Tania, M.B., Antonio, C. and Matlock, T.: Personal WebMelody: Customized Sonification of Web Servers (2001). 